\title{
Time Reversal in Underwater Acoustic Communications
}

\author{
Xiaoping $\mathrm{Li}$ \\ Wright State University, Ohio 45435, \\ United States
}

\author{
Peter Papson \\ University of Alberta, Edmonton, Alberta, Canada \\ T6G 2V4
}

\begin{abstract}
How to achieve high data rate coherent underwater acoustic (UWA) communications is a challenging topic due to several unique properties of UWA environments, especially in shallow water acoustic environments. Time reversal, or phase conjugation in the frequency domain, is a process engages in achieving high data rate and reliable high frequency coherent communications in time-varying UWA environments, while reducing the system complexity significantly compared with traditional methods, such as using large arrays of sensors, or redundantly transmitting at different time. Therefore, timereversal technique has been approved to be a promising technique in future UWA communications and networks. In his paper, we will present an overview of the application of the time-reversal process to acoustic communications. The content includes basic concept and mechanism of timereversal in UWA communications, passive and active timereversal UWA communications, application challenges of the time-reversal technique in UWA communications and networks.
\end{abstract}

\section{General Terms}

Wireless communication and signal processing

\section{Keywords}

Coherent UWA communications; time-reversal technique

\section{INTRODUCTION}

Underwater acoustic (UWA) channels exhibit unique properties of large multi-path propagation delay, several channel fading, large Doppler shift, and fast time variant [1]. Therefore, reliable and high data rate coherent UWA communications have been regarded as one of the most challenging wireless communications.

Modern coherent underwater communications must deal with the inter-symbol interference caused by time-varying multipath environments. The traditional UWA phase coherent communications adopt a decision feedback equalizer with a second order phase lock loop to track phase and mitigate multipath caused inter-symbol interference [2-3]. Recent communication systems are incorporated higher frequencies and different forms of diversity. However, these methods cannot solve the trade-off between system complexity and reliability.

Time reversal, or phase conjugation in the frequency domain, is a process whereby a source at one location transmits sound which is received at another location, time reversed, and retransmitted. The retransmitted sound then returns to its original location [4]. Time-reversal was first used in underwater acoustic communications in 1990s [5].
Time-reversal technique can be divided into two main categories: active time-reversal and passive time-reversal. In the past decades, multi-carrier and multiuser techniques have been widely deployed in the next generation radio frequency wireless communications. In recent years, much literature focuses on applications of Orthogonal Frequency Division Multiplexing (OFDM) and Multi-Input and Multi-Output (MIMO) techniques in UWA communications [6-13]. Therefore, Time-reversal in conjunction with MIMO and OFDM techniques has become a new trend in future high performance UWA communications, especially in complicated fast time-varying shallow water acoustic environments.

In this paper, we proposed an overview of time-reversal techniques in UWA communications. First, we introduce basic concept and working mechanism of time-reversal technique. Then, we provide an overview of both active and passive time-reversal UWA communications. Finally, we illustrate challenging and open issues of applications of timereversal technique in UWA communications and networks.

The remainder of this paper is organized as follows: Section 2 is the introduction of time-reversal UWA communications. Section 3 is the overview of both active and passive time-reversal UWA communications. Section 4 is the discussion of several challenging issues of implementation of time-reversal in UWA communications. Section 5 is the conclusion of this paper.

\section{TIME-REVERSAL TECHNIQUE INTRODUCTION}

Time reversal is a promising UWA communication technique that takes advantage of the reciprocity of the ocean. At the focal point, time reversal compresses dispersive acoustic energy both in time and space. The temporal compression recombines the dispersive multi-path to reduce inter-symbol interference. The spatial focus of the timereversal mirror (TRM) mitigates the effect of channel fading. Therefore, these two unique properties reveal time reversal as a promising technique for UWA communications. The application of time-reversal methods (phase conjugation in the frequency domain) to UWA communications already has been widely investigated in the past decades [14-16]. Let's take the following example to demonstrate the working mechanism of time-reversal UWA communications. 


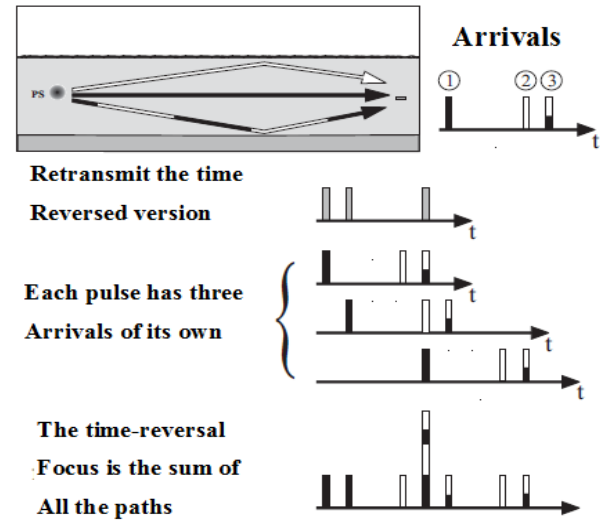

Fig. 1: Schematic description of a time-reversal UWA communication example [14].

In Fig.1 and [17], a probe source (PS) ping is received by a single element some distance away. We assume each path is linear and the destination receives three equal amplitude arrivals representing direct, bottom, and surface reflected paths. Then, the recording is time reversed and retransmitted. Each of the three pulses produces three reciprocal echoes of their own. Eventually, each path adds at the PS position because of linear assumption of waves. [18] revealed relationship between time reversal and linear equalization in wireless communications. Simulation results showed that iterative time reversal could be performed passively, resulting in a procedure that converges to the Moore-Penrose pseudoinverse of the propagation matrix. Especially, [19-20] investigated the performance of time-reversal communications in various UWA channels.

\section{ACTIVE TIME-REVERSAL COMMUNICATIONS}

Active time-reversal communications aim at communicating from an "array" to a "point". By using time reversal, the reflected and refracted waves are converged to the focus point, the signal level received at the focus is increased and the symbol interferences are reduced. However, literature on active time-reversal UWA communications is scarce [21-22]. [21] proposed active time-reversal in combination with adaptive channel equalization and channel coding methods in shallow water environments, results showed that time-reversal processing can further reduce the residual inter-symbol interference (ISI) and cross interference. [22] proposed to combine the focusing effect of time reversal waves and conventional adaptive filter. Simulation results in shallow water environments showed the proposed method could make high speed communication to the long distance possible.

\section{PASSIVE TIME-REVERSAL COMMUNICATIONS}

Passive phase conjugate communications aim at the communicating from a "point" to an "array". In other words, acoustic waves transmitted from a "point" are received at an "array", and the received signals are demodulated by the process which is equal to phase conjugate. Much literature focuses on passive time-reversal communications rather than the active approach. [23] applied single sensor passive TRM to Pattern time delay shift coding scheme (PDS), which could mitigate the ISI caused by multipath channel in shallow water environments and increase the signal propagation distance and available bandwidth. [24] investigated two types of internal wave effects for passive time-reversal communications: one is the rapid channel fluctuation (decreased channel coherence); the other is the long-term (in the scale of hours) performance degradation in the depressed waveguide when the internal waves passed through the acoustic track. [25] proposed a receiver design taking advantage of the time reversal (phase conjugation) and properties of spread spectrum sequences known as Gold sequences to improve link qualities (increase signal-to-noise (SNR) ratio and reduce bit-error-rate (BER) ) of UWA sensor networks. [26] proposed two algorithms to extend the basic passive time reversal technique by adaptively weighting sensor contributions to partially compensate for degraded focusing due to mismatch between the assumed and actual medium impulse responses. [27] presented theoretical performance bounds of time reversal communications via two different approaches: (1) time reversal alone and (2) time reversal combined with channel equalization. By taking advantages of spatial diversity using a number of receivers distributed in space, [28] presented the impact of spatial diversity in passive time reversal communications between a probe source and a vertical receiving array. In 2000, U.S. Navy developed an underwater wireless acoustic network called Seaweb, [29] discussed two modulation schemes and associated receiver algorithms that were developed and tested for Seaweb applications.

\section{CHALLENGING ISSUES OF TIME- REVERSAL COMMUNICATIONS}

Receiver design is one of challenges to achieve timereversal in real UWA communications. A. Song et al. [30] proposed time reversal multichannel receivers using particle velocity channels in vector sensors, results showed that the receiver using vector sensors, results showed this kind of receivers can offer significant size reduction. In addition, they proposed a novel receiver uses time reversal combining followed by a single-channel decision feedback equalizer (DFE) to deal with the ISI for MIMO UWA communications [31].

How to apply time-reversal in other advanced wireless communication techniques is a challenging research topic in recent years. [32-33] proposed two time-reversal applications in multi-channel/OFDM UWA communications. [34-35] proposed two time-reversal applications in MIMO communications in shallow water environments.

Interference cancellation is indispensable to achieve reliable UWA communications. [36] designed a new receiver structure that allows time reversal followed by a single channel decision feedback equalizer, aided by frequent channel updates, which is used to compensate for the time- 
varying ISI. A parallel interference cancellation method is incorporated to suppress the co-channel interference in the MIMO system. [37] proposed a communications receiver architecture composed of a time-reversal approach to suppress multiuser interference followed by an iterative, successive interference cancellation (SIC) scheme to cancel the remaining interference.

\section{CONCLUSION}

The time-reversal technique has become a prominent communication technique in UWA environments, especially for shallow water acoustic environments, due to its low complexity and robust against ISI caused by multi-path propagation. This paper provided a brief overview of the timereversal technique and its applications in UWA communications, which will be a good reference for scientists and engineers in the fields of UWA communications. The discussed challenging issues could be regarded as several open issues of research on time-reversal UWA communications in the future.

\section{REFERENCES}

[1] M. Stojanovic and J. Preisig. "Underwater Acoustic Communication Channels: Propagation Models and Statistical Characterization". IEEE Communication Magazine, pp.84-89, Jan. 2009.

[2] M. Stojanovic, J. A. Capitovic and J. G. Proakis. "Adaptive Multichannel Combing and Equalization for Underwater Acoustic Communicaions". J. Acoust. Soc. Am, vol. 94, pp. 1621-1631, 1993.

[3] M. Stojanovic, J. A. Captovic and J. G. Proakis. "Phase Coherent Digital Communications for Underwater Acoustic Channels". IEEE J. Ocean. Eng. Vol.19, pp.100-111, 1994

[4] G. F. Edelmann, H. C. Song, S. Kim, W. S. Hodgkiss, W. A. Kuperman and T. Akal. "Underwater Acoustic Communications Using Time Reversal". IEEE J. Ocean. Eng. Vol. 30, no. 4, pp. 852-864, 2005.

[5] D. R. Jackson and D. R. Dowling. "Phase Conjugation in Underwater Acoustics". J. Acoust. Soc. Am. Vol. 89, pp.171-181, 1991.

[6] X. Huang. "Capacity Criterion-Based Power Loading for Underwater Acoustic OFDM System with Limited Feedback". IEEE WCNIS Conference, pp.54-58, 2010

[7] X. Huang and V. B. Lawrence. "Capacity CriterionBased Bit and Power Loading for Shallow Water Acoustic OFDM System with Limited Feedback", IEEE $73^{\text {rd }}$ VTC Conference, pp.1-5, 2011

[8] X. Huang and V. B. Lawrence. "Bandwidth-Efficient Bit and Power Loading for Underwater Acoustic OFDM Communication System with Limited Feedback", IEEE $73^{\text {rd }}$ VTC conference, pp.1-5, 2011

[9] C. Polprasert, J. Ritcey and M. Stojanovic. "Capacity of OFDM Systems over Fading Underwater Acoustic Channels". IEEE J. Ocean. Eng., to appear.

[10] X. Huang and V. B. Lawrence. "Effect of WindGenerated Bubbles on OFDM Power Loading for Tim-
Varying Shallow Water Acoustic Channels with Limited Feedback", IEEE Oceans Conference, pp. 1-6, 2011

[11] K. Tuy, D.Fertonani, T.Duman, M. Stojanovic, J. G. Proakis and $\mathrm{P}$. Hursky. "Mitigation of Intercarrier Interference for OFDM over TimeVarying Underwater Acoustic Channels". IEEE J. Ocean. Eng., vol.36, No.2, pp.156-171, 2011

[12] P. Ceballos and M. Stojanovic. "Adaptive Channel Estimation and Data Detection for Underwater Acoustic MIMO OFDM Systems". IEEE J. Ocean. Eng., vol.35, No.3, pp. 635-646, 2010

[13] B.Li, J.Huang, S.Zhou, K.Ball, M.Stojanovic, L.Freitag and P.Willett. "MIMO-OFDM for High Rate Underwater Acoustic Communications". IEEE J. Ocean. Eng., vol.34, No.4, pp.634-645, 2009

[14] G. F. Edelmann, W. S. Hodgkiss, W. A. Kuperman, H. C. Song and T. Akal. "Underwater Acoustic Communication Using Time Reversal”. IEEE Oceans Conference, pp. 2231-2235, 2001

[15] G. F. Edelmann, T. Akal, W. S. Hodgkiss, S. Kim, W. A. Kuperman, and H. C. Song. "An Initial Demonstration of Underwater Acoustic Communication Using Time Reversal". IEEE J. Ocean. Eng. Vol. 27, no. 3, pp.602609,2002

[16] J. Gomes and V. Barroso. "Ray-Based Analysis of a Time-Reversed Mirror for Underwater Acoustic Communication". IEEE ICASSP Conference, pp.29811984,2000

[17] G. F. Edelmann. "An Overview of Time-Reversal Acoustic Communications". TICA Conference, 2005

[18] W. J. Higley, P. Roux and W. A. Kuperman. "Relationship between Time Reversal and Linear Equalization in Digital Communications". J. Acoust. Soc. Am, vol. 120, no. 1, pp. 35-37, 2006

[19] A. J. Silva and S. M. Jesus. "Underwater Communication Using Virtual Time Reversal in a Variable Geometry Channel". IEEE Oceans Conference, pp. 2416-2421, 2002

[20] T. C. Yang. "Relating the Performance of TimeReversal-Based Underwater Acoustic Communications in Different Shallow Water Environments". J. Acoust. Soc. Am. Vol.130, no. 4, pp. 1995-2002, 2011

[21] L. Cai, X. Pan, W. Xu, J. Li and X. Gong. "Underwater Acoustic MIMO Communication Based on Active Time Reversal”. PrimeAisa Conference, pp.45-48, 2009

[22] T. Shimura, Y. Watanabe and H. Ochi. "A Basic Research on the Long Horizontal Active Time Reversal Communication". IEEE Oceans Conference. pp. 22192224, 2004

[23] J. Y, J. Hui, L. Guo and J. Ma. "Channel Equalization Using Passive Time Reversal Mirror in Underwater Acoustic Communication". IEEE ICCT Conference. pp. $1-4,2006$

[24] A. Song, M. Badiey, A. E. Newhall, J. F. Lynch, H. A. DeFerrari, B. G. Katsnelson. "Passive Time Reversal Acoustic Communications through Shallow Water Internal Waves". IEEE J. Acoust. Eng, vol. 35, no.4, pp.756-765, 2010 
[25] M. F. Munir, X. Hong, and F. Filali. "Underwater Acoustic Sensor Networking Using Passive Phase Conjugation". IEEE ICC Conference, pp.2426-2430, 2008.

[26] J. Gomes and A. Silva and S. Jesus. "Adaptive Spatial Combining for Passive Time-Reversed Communications". J. Acoust. Soc. Am. Vol. 124, no, 2, pp.1038-1053, 2008

[27] H. C. Song, W. S. Hodgkiss and S. M. Kim. "Performance Prediction of Passive Time Reversal Communications". J. Acoust. Soc. Am., vol.122, no. 5, pp.2517-2518, 2007

[28] H. C. Song, W. S. Hodgkiss, W. A. Kuperman, W. J. Highley, K. Raghukumar, T. Akal and M. Stevenson. "Spatial Diversity in Passive Time Reversal Communications". J. Acoust. Soc. Am., Vol. 120, no. 4, pp. 2067-2076, 2006

[29] P. Hursky, M. B. Perter, M. Siderius and V. K. McDonald. "Point-to-Point Underwater Acoustic Communications Using Spread Spectrum Passive Phase Conjugation". J. Acoust. Soc. Am., vol. 120, no.1, pp.247-257, 2006

[30] A. Song, M. Badiey, P. Hursky and A. Abdi. "Time Reversal Receivers for Underwater Acoustic Communication Using Vector Sensors". IEEE Oceans Conference, pp.1-10, 2008

[31] A. Song, M. Badiey, V. K. McDonald, T. C. Yang. "Time Reversal Receivers for High Data Rate Acoustic Multiple-Input-Multiple-Output Communication". IEEE J. Ocean. Eng., vol.36, no. 4, pp.525-538, 2011
[32] J. Gomes and V. Barroso. "Time-Reversed OFDM Communication in Underwater Channels". IEEE $5^{\text {th }}$ workshop on signal processing advances in wireless communications,pp.626-630, 2004

[33] J. V. Candy, D. H. Chambers, C. L. Robins, B. L. Guidry, A. J. Poggio, F. Dowla and C. A. Hertzog. "Wideband Multichannel Time-Reversed Processing for Acoustic Communications in Highly Reverberant Environments". J. Acoust. Soc. Am., vol. 120, no. 2, pp. 838-851, 2006

[34] R. Diamant, A. Feuer, and A. A. Dotan. "Time-Reversed MIMO Architecture for Very Shallow Underwater Acoustic Communications". IEEE Oceans Conference, pp.1-5, 2007

[35] H. C. Song, P. Roux, W. S. Hodgkiss, W. A. Kuoerman, T. Akal, M. Stevenson. "Multiple-Input -OutputCoherent Time Reversal Communications in a ShallowWater Acoustic Channel". IEEE J. Ocean. Eng., vol. 31, no. 1 , pp. 170-178, 2006

[36] A. Song and M. Badiey. "Time Reversal MultipleInput/Multiple-Output Acoustic Communication Enhanced by Parallel Interference Cancellation". $J$. Acoust. Soc. Am., vol. 131, no. 1, pp. 281-291, 2012

[37] S. E. Cho, H. C. Song, W. S. Hodgkiss. "Successive Interference Cancellation for Time-Reversed Underwater Acoustic Channels". IEEE Oceans Conference. pp. 1-7, 2009 